\title{
Theoretical and Experimental Investigation on the Probe Design of a Ridge-loaded Slot Type for Near-Field Scanning Microwave Microscope
}

\author{
Hyeok-Woo Son*, Byung-Mun Kim**, Jae-Pyo Hong*** and Young-Ki Cho ${ }^{\dagger}$
}

\begin{abstract}
In this paper, a rectangular waveguide probe with a ridge-loaded straight slot (RLSS) is presented for a near-field scanning microwave microscope (NSMM). The RLSS is located laterally at the end wall of the cavity and is loaded on double ridges in a narrow straight slot to improve the spatial resolution compared with a straight slot. The probe consists of a rectangular cavity with an RLSS and a feed section of a WR-90 rectangular waveguide. When the proposed NSMM is located at distance of $0.1 \mathrm{~mm}$ in front of a substrate without patches or strips, the simulated full width at half maximum (FWHM) of the probe improve by approximately $31.5 \%$ compared with that of a straight slot without ridges. One dimensional scanning of the E-plane on a sample under test was conducted, and the reflection coefficient of the near-field scanning probe is presented.
\end{abstract}

Keywords: Rectangular waveguide probe, Ridge-loaded straight slot, Near-field scanning, Microwave microscopy

\section{Introduction}

Microwave scanning probes for near-field scanning microwave microscopes (NSMMs) and for nondestructive evaluation have recently attracted much interest [1-8]. However, the resolution of a microwave imaging system is limited by the wavelength because of the diffraction characteristic of waves. NSMM can resolve objects much smaller than the wavelength of the excited wave using the characteristic of an evanescent electromagnetic wave [1]. In the reactive near-field region of an antenna, the field is non-radiated and is localized near the radiator, which is also called an evanescent wave region. The power density can be seen to be totally reactive (purely imaginary). The energies of the magnetic and electric fields are stored. Therefore, this method can be used to generate evanescent waves and to implement a microwave microscopic imaging system. The distance between the sample under test (SUT) and the probe should be as near as possible to ensure that the SUT is in the reactive near-field region, and the size of the probe should be small to achieve higher resolution.

Microwave near-field probes are either broadband or resonant. Broadband probes typically use electromagnetic waveguides, whereas resonant probes normally employ a resonant cavity that is coupled through a sub-wavelength-

$\dagger \quad$ Corresponding Author: School of Electronics Engineering, Kyungpook National University, Korea. (ykcho@ee.knu.ac.kr)

* School of Electronics Engineering, Kyungpook National University, Korea. (hwson@ee.knu.ac.kr)

** Division of IT Cooperative Systems, Gyeongbuk Provincial College, Korea. (kimbyte@gpc.ac.kr)

*** Department of Electronics Engineering, Kyungil University, Korea. (jphong@kiu.kr)

Received: March 10, 2015; Accepted: May 7, 2015 sized probe. Resonant probes, which employ resonant types of slots [9], are more sensitive because the signal-tonoise ratio in a resonator increases with the resonator quality factor $(\mathrm{Q})$. Thus, they are very efficient in the narrow frequency band for which they are designed. This increase in sensitivity and field strength is accompanied by a narrower frequency band, where the drop in the amplitude depends on Q, which results from the shift in the resonant frequency under different SUT environments [10]. Four independent research groups have designed different resonant probes and have published their works in close succession to one another since 1993 [3-7]. In the present study, a rectangular waveguide probe with a straight slot is designed for a microwave microscope $[11,12]$. To improve the spatial resolution for a straight slot, a ridge-loaded straight slot (RLSS) at the end-side wall of a waveguide is presented as a near-field scanning probe. The proposed probe consists of a rectangular waveguide cavity with a RLSS and a feed section of a WR-90 rectangular waveguide. If the length of the cavity and the windowwidth of the inductive iris are properly adjusted to realize a matching method, the cavity is critically coupled to the slot of the probe while pointing at the substrate of a printed circuit board (PCB). Thus additional screw tuner does not need to be used for fine tunning [11].

The characteristics of the probe, such as the spatial resolution i.e., full width at half maximum (FWHM) and reflection coefficient, are examined using a commercial electromagnetic simulator Ansoft HFSS and compared with those of a straight slot without ridges, which has been conventionally used in waveguide probes $[11,12]$. Using a reactive near-field generated by the slot, this probe is located near the SUT and measures the reflection coefficient 
using a vector network analyzer (VNA) when the location of the probe is varied. In this study, one-dimensional scanning is performed, and the measurement results of a near-field scanning waveguide probe for a PCB with seven $0.5 \mathrm{~mm}$ wide metallic strips and three metallic patches with a gap $0.5 \mathrm{~mm}$ gap are presented.

\section{Electric Field Characteristics for an RLSS}

In the developed NSMM, a rectangular waveguide probe is designed as a near-field scanning probe. Fig. 1 (a) shows the block diagram of the conventional rectangular waveguide cavity probe with screw tuner for near-field scanning and Fig. 1 (b) shows the block diagram of the proposed rectangular waveguide cavity probe without screw tuner. The proposed probe consists of a rectangular waveguide cavity with a narrow RLSS and a rectangular waveguide feed section. The cavity is coupled through an inductive iris for probe matching. By adjusting the window-width $w_{\text {in }}$ of the inductive iris at Fig. 2, this probe can excite a specific resonant mode in the RLSS of the cavity. The resonant length $l_{c}$ of the cavity for the dominant mode $T E_{101}$ is influenced by the admittance of the slot and the inductive iris, and is slightly smaller than $0.5 \lambda_{g}$ (guided wavelength). If the slot is located at the center of the end-side wall and the direction of the slot is along the $\mathrm{x}$-axis direction, the slot can block the current on the surface of the end-side wall. Near-field enhancement can be observed in the ridge gap area of the proposed straight slot with double ridges. The currents induced on the double ridges of the slot are guided toward the terminals. Charges accumulate at the terminals, which leads to a displacement current across the gap because the

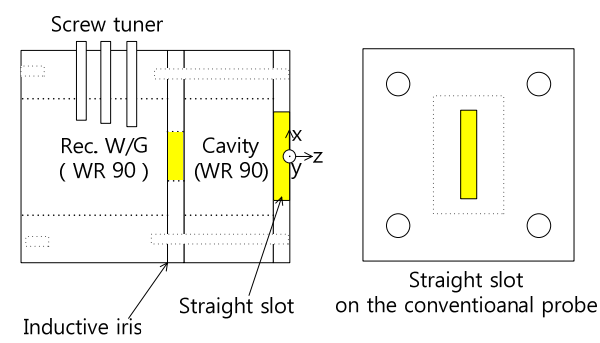

(a) conventional probe

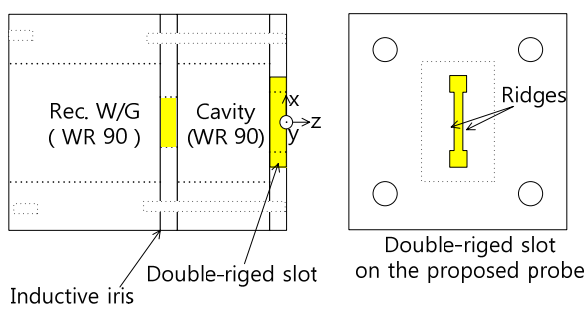

(b) proposed probe

Fig. 1. Block diagram of the rectangular cavity probes for NSMM

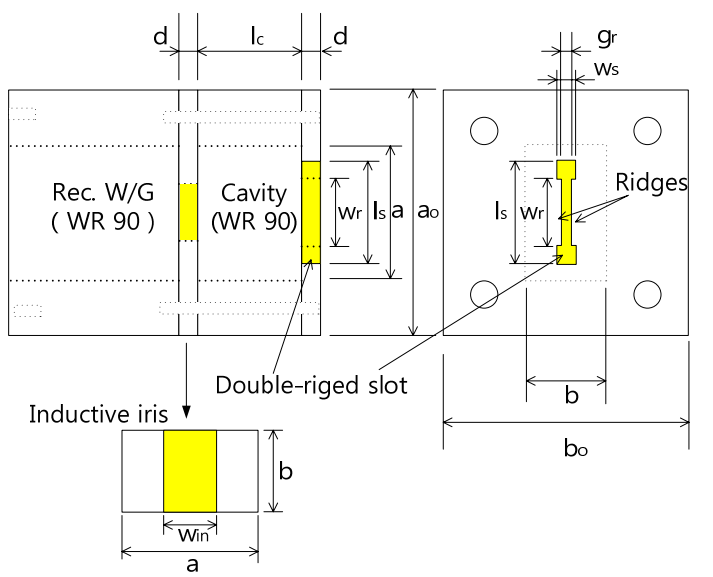

Fig. 2. Structure diagram of the proposed probe for NSMM

terminals are open circuited. This displacement current radiates similar to a Hertzian dipole with length $w_{r}$ [8]. The field distribution in the ridge gap area strongly depends on the width and gap of the ridges. The field at the E-plane edges of the RLSS is enhanced compared with that in the center. This edge enhancement is more pronounced in wider slots, although it occurs only very close to the slot and decays rapidly with the distance. This enhancement originates from the electric field concentration at the sharp conducting edges of the slot $[12,13]$. Therefore, when the SUT is in the vicinity of the slot, its evanescent near field is also quite different due to the SUT environment.

\section{Design of a Rectangular Waveguide Probe}

When the proposed probe is located in front of the SUT as shown in Fig. 3, the normalized input admittance, $y_{\text {in }}$ $(z=-d)$ would be those shown in Fig. 4 . The distance, $d_{s}$ between the probe and the SUT is $0.1 \mathrm{~mm}$. Table 1 lists the

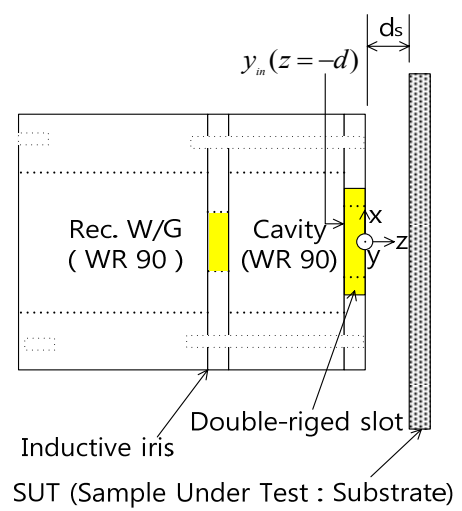

Fig. 3. Waveguide probe and substrate SUT

Table 1. Dimensions of the proposed probe

\begin{tabular}{c|c|c|c|c|c|c}
\hline Parameters & $a$ & $b$ & $l_{c}$ & $d$ & $l_{s}$ & $w_{s}$ \\
\hline Values $(\mathrm{mm})$ & 22.86 & 10.16 & 18.27 & 1.00 & 14.50 & 1.00 \\
\hline Parameters & $w_{r}$ & $g_{r}$ & $w_{i n}$ & $a_{0}$ & $b_{0}$ & $d_{s}$ \\
\hline Values $(\mathrm{mm})$ & 12.50 & 0.50 & 7.90 & 40.00 & 40.00 & 0.10 \\
\hline
\end{tabular}


NSMM parameters. The used waveguide is a WR-90 waveguide (width $a=22.86 \mathrm{~mm}$; height $b=10.16 \mathrm{~mm}$ ) and FR-4 $\left(\varepsilon_{r}=4.3\right.$, thickness $\left.=1.57 \mathrm{~mm}\right)$ is used as the SUT.

The normalized input admittance, $y_{\text {in }}$ at desired frequency $f_{0}(10.40 \mathrm{GHz})$ is $2.1843+j 9.6284$. At Fig. 5(a),

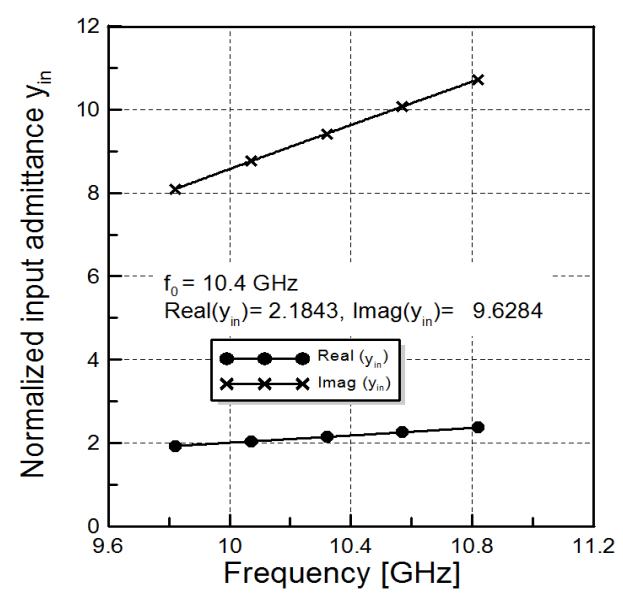

Fig. 4. SUT Normalized input admittance of the probe in front of the substrate, $d_{s}=0.1 \mathrm{~mm}$.

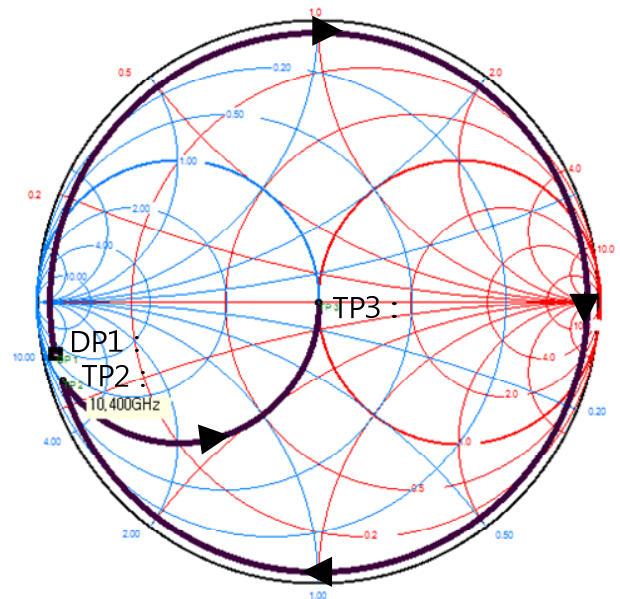

(a)

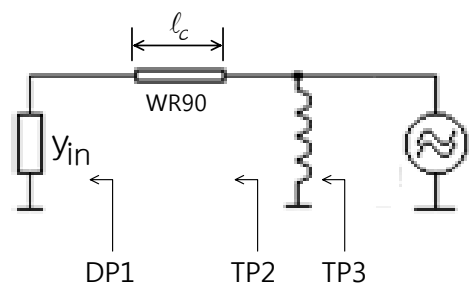

\begin{tabular}{|c|c|c|}
\hline Start & Point & $\mathbf{y}$ (Normalized admittance) \\
\hline$\square$ & DP1 & $2.1843+\mathrm{j} 9.6284$ \\
\hline & TP2 & $0.9844+\mathrm{j} 6.5125$ \\
\hline & TP3 & $0.9844+\mathrm{j} 0.0$ \\
\hline
\end{tabular}

(b)

Fig. 5. Impedance matching for the normalized input admittance of the probe: (a) Impedance transformation in Smith chart; (b) Schematic diagram which displayed for the network on Smith chart.
DP1 on Smith chart is the first data point of $y_{i n}$. When the length of the rectangular waveguide (the cavity : WR 90) is properly adjusted, the real part of the normalized input admittance $\operatorname{Re}\left[y_{\text {in }}\left(z=-\left(d+l_{c}\right)\right]\right.$ is approximately " 1 " like as TP2 on Smith chart. The $y_{\text {in }}$ is $0.9844+\mathrm{j} 6.5125$ when the length of the waveguide $l_{c}$ is $0.492 \lambda_{g}(18.27 \mathrm{~mm})$. To realize a matching condition, which means canceling out the capacitive component at the location $l_{c}$ of the proposed probe, a shunt inductive iris $\left(w_{i n}=8.50 \mathrm{~mm}, d=1.0 \mathrm{~mm}\right)$ is placed at $z=-\left(d+l_{c}\right)$ in the rectangular waveguide, as shown in Fig. 2. In Fig. 5(b), the schematic diagram displays the network on Smith chart for the impedance matching of the proposed probe.

Fig. 6 shows the normalized susceptance $b_{\text {ind }}$ and conductance $g_{\text {ind }}$ according to window-width $w_{\text {in }}$ in the WR-90 waveguide. At window-width $w_{i n}<10 \mathrm{~mm}$ and thickness of the iris $d=1.0 \mathrm{~mm}$, the iris operates as a transformer because the normalized conductance $g_{\text {ind }}$ is not " 1 " under this condition. Thus, the resonance frequency of the input admittance of the NSMM is shifted near 10.32 GHz. Further window-width $w_{\text {in }}$ is adjusted to $7.90 \mathrm{~mm}$ to realize a matching condition. The reflection coefficients

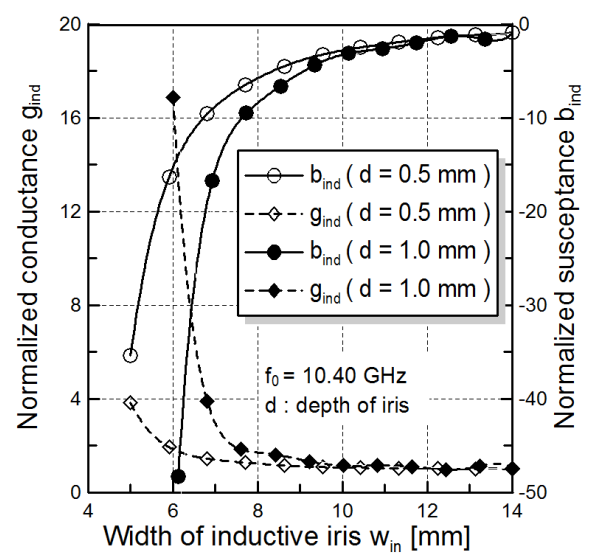

Fig. 6. Normalized admittance of the inductive iris for the variation of window-width

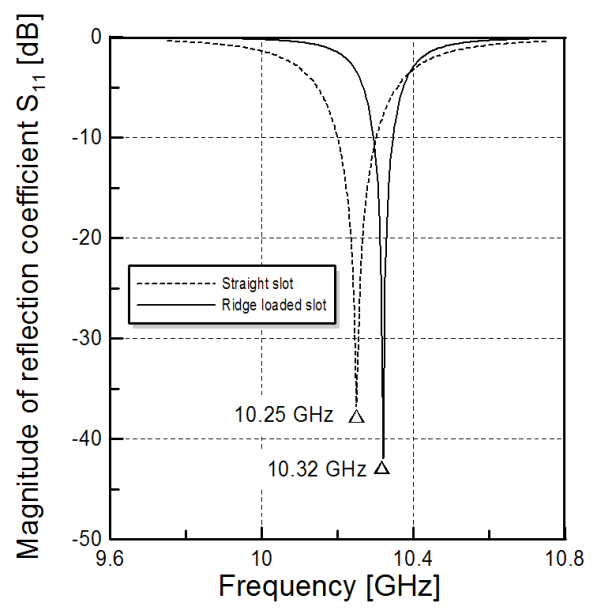

Fig. 7. Measured reflection coefficient $S_{11}$ of the probes pointing at the substrate 


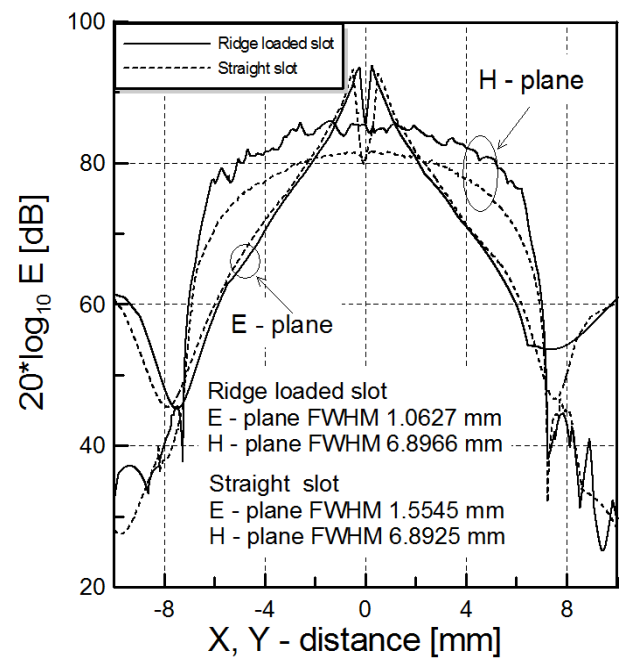

Fig. 8. Electric field intensity distribution on the SUT surface. $\left(d_{s}=0.1 \mathrm{~mm}\right)$

of the proposed probe with RLSS are compared with those of the probe with a straight slot, as shown in Fig. 7. The distance between the probes and the substrate without obstacles is $0.1 \mathrm{~mm}$. The resonant frequency of the probe with a straight slot is $10.25 \mathrm{GHz}$, and the length of the waveguide cavity $l_{c}$ is $18.27 \mathrm{~mm}$. This length is designed to be the same as that of the proposed probe with RLSS. The inductive iris window-width $w_{i n}$ should be $9.09 \mathrm{~mm}$.

Fig. 8 shows that the electric field intensity on the SUT has a peak value around the E-plane edge of the straight slot where the incident power is $1 \mathrm{~W}$ and $d_{s}=0.1 \mathrm{~mm}$. We note that the electric field intensity of the RLSS is strongly concentrated on the surface of the SUT compared with that in the straight slot. The FWHM of the H-plane is approximately $6.89 \mathrm{~mm}$ in both cases. In the E-plane case, the FWHM of the straight slot is $1.5545 \mathrm{~mm}$, whereas that of the RLSS is $1.0627 \mathrm{~mm}$. The spatial resolution of the RLSS has improved to approximately $31.51 \%$.

\section{Experimental results}

Fig. 9 shows the experimental arrangement of the NSMM. In this study, the SUTs are made of a PCB with different patterns of metallic strips and patches. The moving controller of the experimental system is a cross roller stage (MAU XY 8030-C-N), Toptek with the movement accuracy of $\pm 30 \mu \mathrm{m}$. An Agilent E8364B VNA is used to measure the reflection coefficient of the probe. The first sample is a PCB with seven $0.5 \mathrm{~mm}$ wide metallic strips as shown in Fig. 10. After positioning the probe with gap $d_{s}=0.1 \mathrm{~mm}$ between the PCB and the probe when the probe points at the substrate portion of the PCB without strip, the measured frequency responses of the probe are in resonant modes at $10.261 \mathrm{GHz}$ (straight slot case) and $10.330 \mathrm{GHz}$ ( ridge-loaded slot case).

Fig. 11 shows the measured reflection coefficients at the

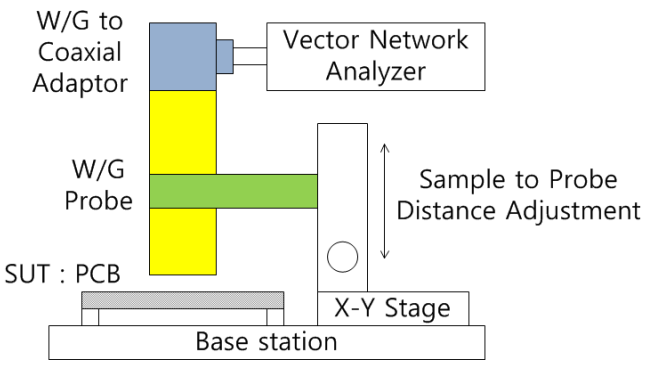

Fig. 9. Experimental equipment arrangement

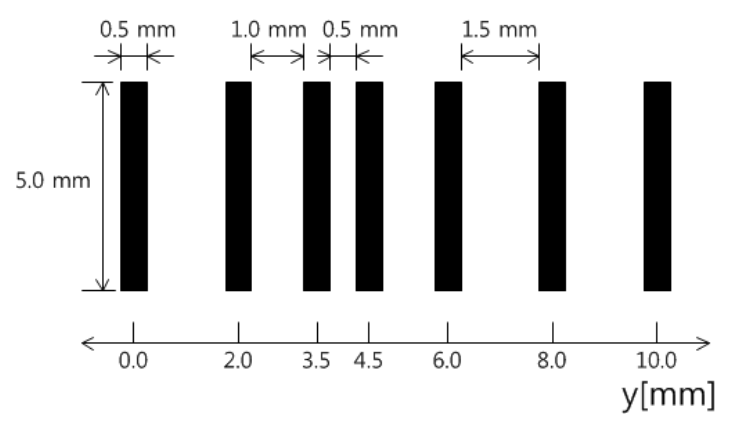

Fig. 10. Layout of the PCB with seven wide strips

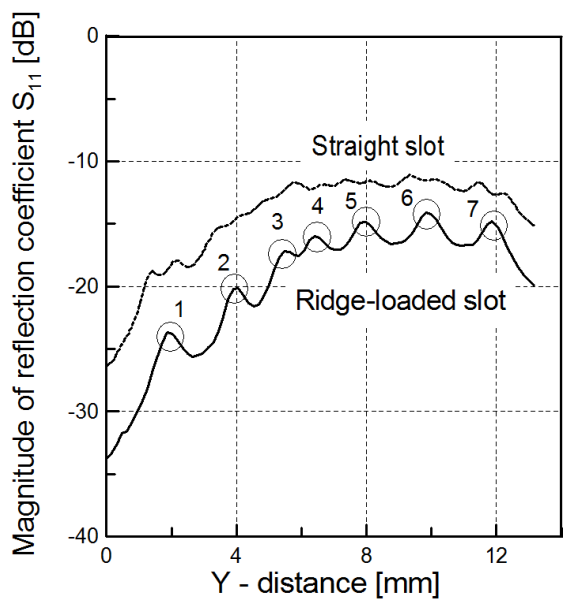

Fig. 11. Measurement results for the SUT in Fig. 10 at resonant frequencies of conventional and proposed probe. : Straight slot : 10.261 and RLSS :10.330 GHz.

two resonant frequencies (Straight slot : 10.261 and RLSS : $10.330 \mathrm{GHz}$ ) while the probe is moving in y-axis direction. From these measurement results, we can find that the probe with the RLSS can resolve the two lines at Nos. 3 and 4 in which the gap between these two strips is only $0.5 \mathrm{~mm}$ and the dimension of the gap is almost the same as that of the ridged slot. When the probe is located over the strip, the measured reflection coefficient achieves a peak, and Fig. 10 shows that it has seven peaks at the locations of 2.0, 4.0, 5.5, 6.5, 8.0, 10.0, and $12.0 \mathrm{~mm}$, which agree with the locations of the strips. Fig. 11 shows that the other probe with a straight slot has a difficulty resolving these two strips at Nos. 3 and 4 because the slot 


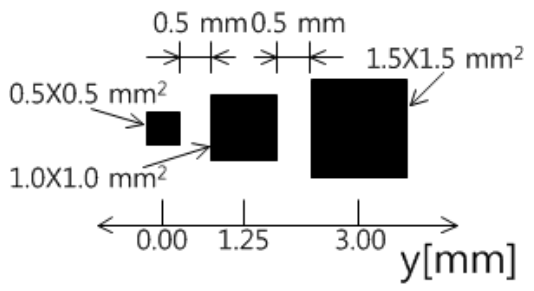

Fig. 12. Layout of the PCB with three metallic patches $(0.5$ mm gap)

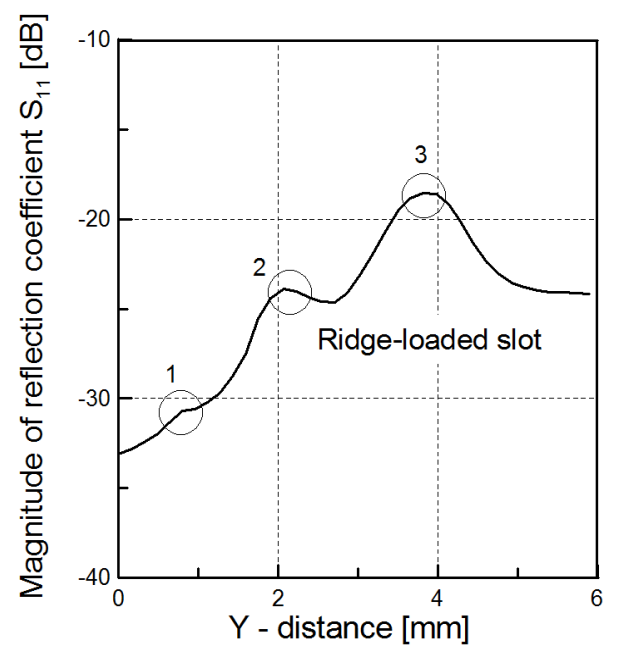

Fig. 13. Measurement results for the SUT in Fig. 12 at resonant frequency of $10.330 \mathrm{GHz}$

width $\left(w_{s}=1.0 \mathrm{~mm}\right)$ is wider than the gap between these two strips. The second sample is a PCB with three metallic patches with a $0.5 \mathrm{~mm}$ gap, as shown in Fig. 12. The areas of the three patches are $0.5 \times 0.5 \mathrm{~mm}^{2}, 1.0 \times 1.0 \mathrm{~mm}^{2}$, and $1.5 \times 1.5 \mathrm{~mm}^{2}$. The measured frequency responses of the proposed probe are in a resonant mode at $10.330 \mathrm{GHz}$.

Fig. 13 shows that the locations of the three peaks 1.0, 2.0 , and $4.0 \mathrm{~mm}$ agree with those of the patches. However, the first patch is quite difficult to distinguish because the patch height is small. In the present ridged aperture, the desired spatial confinement of the electromagnetic energy for better resolution can be achieved by reducing the transverse length (along $\mathrm{x}$-axis) of the ridged aperture. This length reduction along $\mathrm{x}$-axis is the decisive role in improving the H-plane resolving power of the probe [14].

\section{Conclusion}

In this study, a microwave microscope system that uses a rectangular waveguide cavity probe has been developed. A RLSS at the end-side wall of a cavity is implemented to enhance the spatial resolution as a near-field scanning probe. To eliminate additional tuners such as a screw tuner, we can select the width of the inductive iris and the length of the cavity for the best resolution to measure the geometric characteristics of the SUT while pointing at the substrate of the PCB. From the measurement results, the geometric structures of the SUT can be identified from the measured reflection coefficient. This method can potentially image the geometric structures of the metallic patterns as a contactless and non-destructive testing tool. Further research in this area will be performed to improve the H-plane spatial resolution of a metallic patch with a small area; therefore, studies on the ridge structure of straight slots will be needed.

\section{Acknowledgement}

This study was supported by the Basic Science Research Program through the National Research Foundation of Korea (NRF) funded by the Ministry of Education, Science and Technology (NRF-2010-0024647) and by Kyungpook National University Research Fund, 2012

\section{References}

[1] E. A. Ash and G. Nicholls, "Super-resolution aperture scanning microwave microscope," Nature, vol. 237, pp. 510-512, 1972.

[2] N. N. Qaddoumi, M. Abu-Khousa and W. M. Saleh, "Near-field microwave imaging utilizing tapered rectangular waveguides," IEEE Trans. Instrum Meas., vol. 55, no. 5, pp.1752-1756, Oct. 2006.

[3] HM. T. Azar, J. L. Katz, and S. R. LeClair, "Evanescent microwaves: a novel super-resolution noncontact nondestructive imaging technique for biological applications," IEEE Trans. Instrum Meas., vol. 48, no. 6, pp. 1111-1116, Dec. 1999.

[4] R. S. Aga, X. Wang, J. R. Dizon, J. Noffsinger and J. $\mathrm{Z}$. Wu, "Application of near-field scanning microwave probe to current density mapping in conducting films at room temperature," Appl. Phys. Lett., vol. 86, pp. 234101, 2005.

[5] D. E. Steinhauer, C. P. Vlahacos, S. K. Dutta, F. C. Wellstood and S. M. Anlage, "Surface resistance imaging with a scanning near-field microwave microscope," Appl. Phys. lett., vol. 71. no. 12, pp. 1736-1738, 1997.

[6] C. Gao, T. Wei, F. Duewer, Y. Lu and X. D. Xiang, "High spatial resolution quantitative microwave impedance microscopy by a scanning tip microwave near-field microscope," Appl. Phys. lett., vol. 71, no. 13, pp. 1872-1874, 1997.

[7] M. Abu-Teir, M. Golosovsky, D. Davidov, A. Frenkel and H. Goldberger, "Near-field scanning microwave probe based on a dielectric resonator," Rev. Sci. Instr., vol. 72, no. 4, pp. 2073-2079, 2001.

[8] B.M. Kim, K.P. Hwang and Y.K. Cho, "Investigation of a planar bow-tie antenna fed by a flanged rectangular waveguide," in Proceeding of APS-URSI 
2011 Conference, Spokane, American, July 2011.

[9] Young-Ki Cho, Kun-Woo Kim, Ji-Hwan Ko and Jong-Ig Lee, "Transmission Through a Narrow Slot in a Thick Conducting Screen," IEEE Trans. Antennas Propag., vol. 57, no. 3, pp.813-816, 2009.

[10] Rosner B.T. and van der Weide D.W., "Highfrequency near-field microscopy," Rev. Sci. Instr., vol. 73, no. 7, pp. 2505-2525, 2002.

[11] Sung-Niem Hsich, Tah-Hsiung Chu, and Ming-Tang Chen, "Scanning Near-Field Microwave Microscope Using a Rectangular Waveguide Probe with Different Resonant Modes of Cavity," in Proceedings of the Asia-Pacific Microwave Conference 2011, Melbourne, Australia, Dec. 2011.

[12] M. Golosovsky, E. Maniv, D. Davidov, A. Frenkel, "Near-field of a scanning aperture microwave probe: a 3-D finite element analysis," IEEE Trans. Instrum. Meas., vol. 51, no. 5, pp.1090-1096, 2002.

[13] Y. Leviatan, "Study of near-zone fields of a small aperture," J. Appl. Phys., vol. 60, no. 5, pp.1577-1583, 1986.

[14] B. M. Kim, H. W. Son, J. P. Hong and Y. K. Cho, "A novel epsilon near zero tunneling circuit using double - ridge rectangular waveguide," J. Electromagn. Eng. Sci., vol. 14, no. 1, pp. 36-42, 2014.

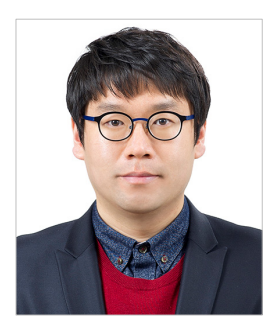

Hyeok-Woo Son $\mathrm{He}$ was born in Korea in 1983. He received his B.S. and M.S. degree from the Department of Electronics Engineering of Kyungpook National University, Daegu, Korea, in 2009 and 2011, respectively. $\mathrm{He}$ is currently taking his Ph.D at Kyungpook National University. His current research interests are electromagnetic theory, metamaterial, antenna design and MRI RF Coil design.

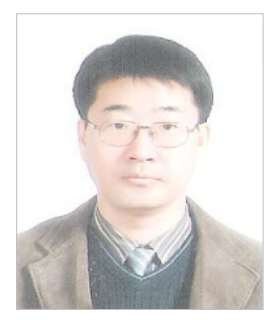

Byung-Mun Kim He was born in Waegwan, Korea. He received the B.S. degree in electronic engineering from Kyungpook National University, Daegu, Republic of Korea, in 1986, and the M.S. and Ph.D. degree in electronic Engineering from Kyungpook National University, in 1988 and 2015, respectively. Since 1997, he is a Professor of Department of IT Cooperative Systems of Gyeongbuk Provincial College, Yecheon, and his research areas are antenna and microwave circuits, and radar system.

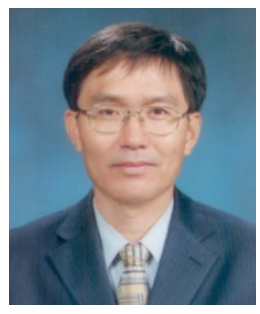

Jae-Pyo Hong He was born in Daegu, Korea. He received the B.S. degree in electronic engineering from Kyungpook National University, Daegu, Republic of Korea, in 1981, and the M.S. and Ph.D. degree in electronic Engineering from Kyungpook National University, in 1983, and 1989, respectively. Since 1990, he is a Professor of electronic engineering of Kyungil University, Kyungsan, and his research areas are antenna and microwave circuits design.

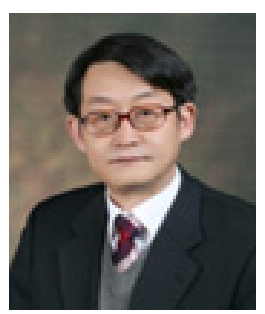

Young-Ki Cho $\mathrm{He}$ received a B.S. degree in Electrical Engineering from Seoul National University, Seoul Republic of Korea, in 1978. He received M.S. and Ph.D. degrees in Electrical Engineering from the KAIST (Korea Advanced Institute of Science and Technology), Daejon, Korea. In 1981, he joined the School of Electrical Engineering and Computer Science at Kyungpook National University, Daegu Korea. Since 1992 he has been Korea's representative on URSI Commission B, Fields and Waves. In 2008 he became President of the Korean Institute of Electromagnetic Engineering and Science. His research interests include electromagnetic theory, scattering problem, antenna, and RF devices. 